\title{
EPR characterization of vanadium dopant sites in DUT-5(Al)
}

\author{
Kwinten Maes ${ }^{a}$, Irena Nevjestića ${ }^{a}$ Hannes Depauw ${ }^{b}$, Karen Leus ${ }^{b}$, Pascal Van Der Voort ${ }^{b}$, Henk \\ Vrielinck $^{\mathrm{a}}$ and Freddy Callens ${ }^{\mathrm{a}}$ \\ aDepartment of Solid State Sciences, Ghent University, Krijgslaan 281-S1, B-9000 Gent, \\ Belgium. E-mail: henk.vrielinck@ugent.be

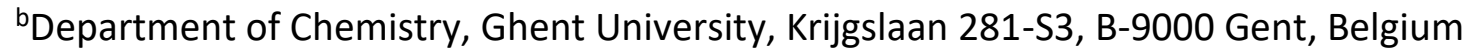

\begin{abstract}
The structural environment of the $\mathrm{V}^{\mathrm{IV}}$ ions doped in the metal-organic framework (MOF) DUT-5(AI) ((AI'"IOH)BPDC) was studied with electron paramagnetic resonance (EPR). At low $\mathrm{V}$ concentrations, two different vanadyl components are observed in the EPR spectrum: a broad-line component, best visible at ambient air conditions, and a narrow-line component, becoming more prominent at lower pressures. Recording the EPR spectra at both X- and Qband, spin-Hamiltonian parameters were derived for both components. Similarities between the spectrum of the narrow-line component and the spectrum of $\mathrm{V}^{\mathrm{IV}}$ centers in the large pore state of a related MOF, MIL-53(AI) ((AI'"IOH)BDC), are discussed, including their sensitivity to oxygen. The broad-line component cannot conclusively be identified by comparison with $\mathrm{V}^{\mathrm{IV}}$ centers in MIL-53(Al). Increasing the concentration of $\mathrm{V}^{\mathrm{IV}}$ in the DUT5(Al) framework, a third vanadyl component appears in the spectrum, together with a broad feature related to the spectrum of COMOC-2(V) (( $\left.\left.V^{I V}=0\right) B P D C\right)$. Surprisingly, this third component appears to be linked with a narrow pore state of the mixed-metal MOF.
\end{abstract}

Keywords: Metal-organic frameworks (MOFs); breathing; oxygen sensitivity; flexibility; electron paramagnetic resonance (EPR)

\section{Introduction}

Metal-organic frameworks (MOFs) are porous crystalline materials constructed of metalinorganic units connected with organic linkers. Their structural characteristics, like crystal structure, pore shape and size, and physicochemical properties can to some extent be steered by the choice of these two building blocks. This tuneability gives MOFs a competitive advantage over other porous materials, like zeolite frameworks or activated carbon. Various applications for MOFs have been demonstrated or are currently being explored, e.g. in catalysis, sensing, gas storage, separation and adsorption [1-4].

Within the wide variety of existing MOFs, the MIL-53 family (MIL = Matériaux de l'Institut Lavoisier), built from infinite $\mathrm{M}^{\text {III }}(\mathrm{OH}$ ) chains ( $\mathrm{M}=\mathrm{Al}, \mathrm{Sc}, \mathrm{V}, \mathrm{Cr}, \mathrm{Fe}$, or $\mathrm{Ga}$ ) [5-10] connected by 1,4-benzenedicarboxylate (BDC) linkers, has received considerable research attention over the past 15 years.

For some of these structures $(\mathrm{M}=\mathrm{Al}, \mathrm{V}, \mathrm{Cr}, \mathrm{Ga})$, the framework can reversibly change between a state with open, large pores (labelled here as Ip) and a state with closed, narrow pores (np), a property generally referred to as "breathing". These transitions can be induced by external triggers, like temperature, mechanical pressure and exposure to (and the 
presence of) certain gasses, most notably water vapor and $\mathrm{CO}_{2}$. After solvothermal synthesis, the as synthesized structures (as) do not immediately exhibit breathing. First solvent and excess linker molecules that block the pores need to be removed by solvent extraction or calcination (activation of the MOF). Within this series of breathing MOFs MIL53(V) takes a special position: when activated by solvent extraction, it exhibits breathing, but upon calcination, the metal-inorganic backbones transform into vanadyl $\left(\mathrm{V}^{\mathrm{IV}}=\mathrm{O}\right)$ chains, and the framework adopts a rigid Ip structure, that was labelled MIL-47 [11].

In an attempt to better understand the mechanism of the Ip to np structural transitions and to tune the breathing process, we studied MIL-53(Al) doped with V [12-14]. We found that the electron paramagnetic resonance (EPR) spectrum of vanadyl defects (with effective electron spin $\mathrm{S}=1 / 2$ ), replacing regular $\mathrm{Al}-\mathrm{OH}$ nodes in the framework, is a very sensitive marker for the framework state. By monitoring X-ray diffraction (XRD), Fourier Transform Infrared (FTIR), and multifrequency EPR spectroscopy in situ during breathing transformations, we were able to isolate and completely characterize the EPR spectra for $\mathrm{V}^{\mathrm{IV}}=\mathrm{O}$ defects in 4 different states of the MIL-53(Al) framework, as shown in Figure 1 at a microwave frequency of $34 \mathrm{GHz}$ (Q-band). In Table 1 the spin-Hamiltonian parameters for $\mathrm{V}^{\mathrm{IV}}=\mathrm{O}$ centers in these four framework states are summarized.

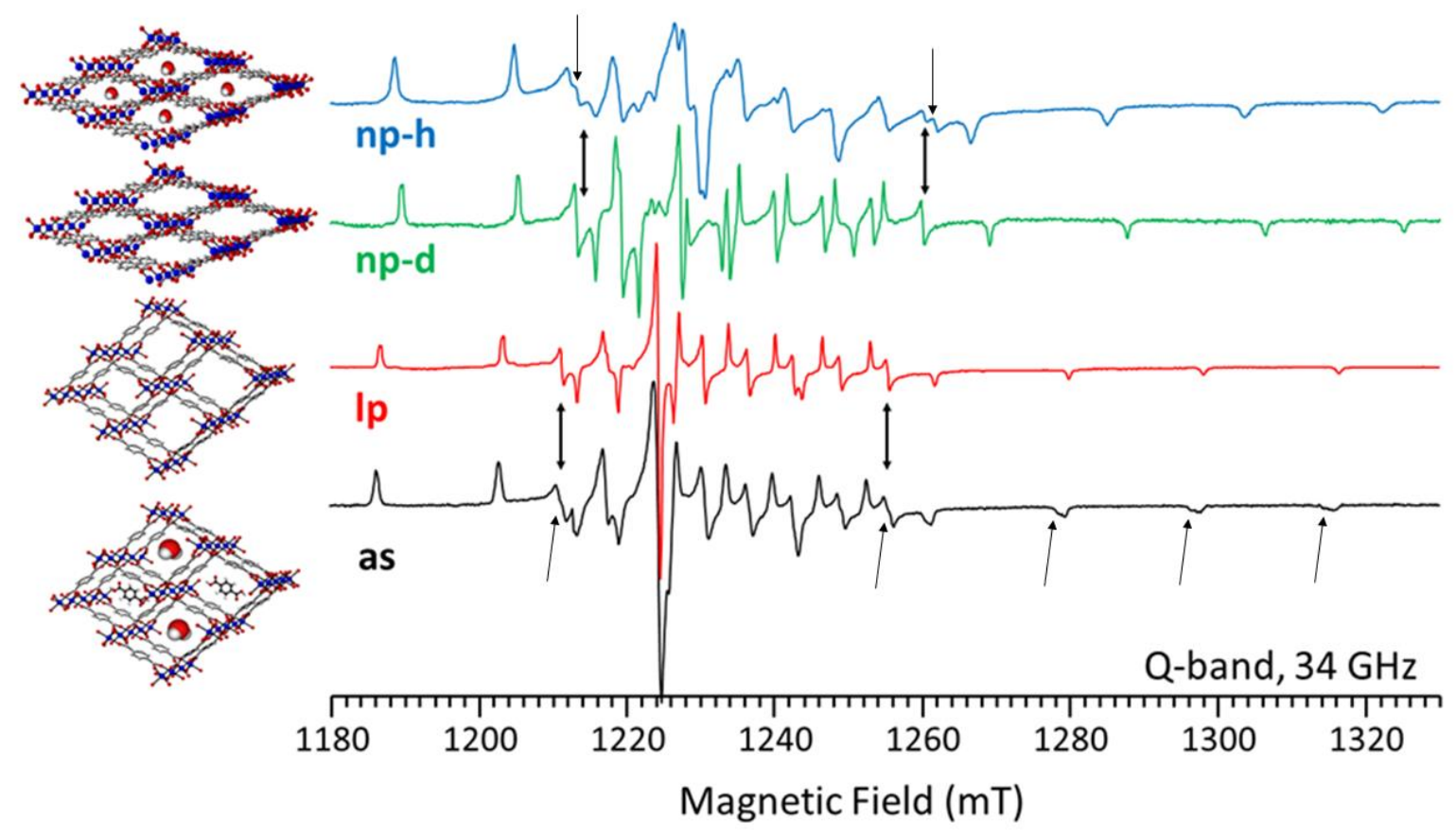

Figure 1: Experimental Q-band EPR spectra of V-doped MIL-53(Al) showing its four different framework states: hydrated narrow pore ( $\boldsymbol{n p}$ - $\boldsymbol{h})$, dehydrated narrow pore (np-d), large pore (Ip) and as synthesized (as). On the left, next to each EPR spectrum, is a graphical representation of the structure, showing its pore structure and the presence of molecules in its pores. Arrows indicate the positions in the spectra where additional features appear for the filled pore structures, in comparison with the empty pore structures.

When stored at room temperature (RT) and ambient air atmosphere, activated MIL-53(AI) adopts a hydrated $\mathbf{n p}$ framework state (np-h). Our study has shown that upon heating, this framework dehydrates (np-d) before making the transition to the Ip state. A detailed analysis of the EPR spectra further revealed that the Ip and $\mathbf{n p - d}$ (empty) framework states 
exhibit only one site for the $\mathrm{V}^{\mathrm{IV}}=\mathrm{O}$ defects. In contrast, the $\mathrm{V}^{\mathrm{IV}}=\mathrm{O}$ EPR spectra for the $\mathbf{n p}-\mathbf{h}$ and as states reveal that at least two distinct dopant sites exist, as indicated by arrows in Figure 1. Similar conclusions on inequivalence of the metal sites in these two states were obtained from magic angle spinning nuclear magnetic resonance studies [15, 16]. Finally, exposure of the MOF to $\mathrm{O}_{2}$ (paramagnetic), either pure or in air, led to a marked EPR linewidth increase for frameworks in the Ip state, but had virtually no effect for the three other states. This indicates that only in the Ip state $\mathrm{O}_{2}$ can enter the framework and broaden the $\mathrm{V}^{\mathrm{IV}}=\mathrm{O}$ EPR spectrum through exchange interactions.

Recently, we started to study a second family of MOFs. They have the same topology as MIL53(Al) - MIL-47(V), with the same metal-inorganic building blocks, but biphenyl-4,4'dicarboxylate (BPDC) as linkers. (AI"IOH)BPDC, also called DUT-5(AI) (DUT = Dresden University of Technology) [17], has a rigid, Ip framework and does not show a breathing effect. In ( $\left.V^{I V}=0\right) B P D C$, i.e. COMOC-2(V) (COMOC = Centre for Ordered Materials, Organometallics and Catalysis, Ghent University) [18], we recently detected a new type of $\mathrm{CO}_{2}$-triggered breathing. Only part of the framework changes state as a function of $\mathrm{CO}_{2}$ pressure, while the rest remains in the original Ip state. The "breathing" part of the framework first transforms to a $\mathbf{n p}$ state and then opens again at higher pressures to a Ip state that is markedly different from the original. In mixed $\left(A I^{I I I} O H\right)_{\times}\left(V^{I V}=O\right)_{1-x} B P D C M O F s$, an EPR spectral component was observed that showed similar characteristics as $\mathrm{V}^{\mathrm{IV}}=\mathrm{O}$ in Ip MIL53(Al), but also other components were found [19]. In the present paper, we further explore the similarities and remarkable differences in EPR characteristics for $\mathrm{V}^{\mathrm{IV}}=\mathrm{O}$ in these two families of MOFs. We focus first and foremost on the spectrum analysis of DUT-5(AI), doped with low concentrations of $\mathrm{V}$, followed by an exploratory study of the spectra of higher doped samples.

\section{Experimental}

2.1 Synthesis of the mixed-metal MOF and XRD characterization

DUT-5(Al) doped with various concentrations of $\mathrm{V}^{\mathrm{IV}}$ has been synthesized in accordance with Depauw et al. [19]. A mixture of Dimethylformamide (DMF), $\mathrm{H}_{2} \mathrm{BPDC}$ and different ratios of the metal salt vanadium oxide sulphate $\left(\mathrm{VOSO}_{4} \bullet \mathrm{H}_{2} \mathrm{O}\right)$ and aluminium nitrate $\left(\mathrm{Al}\left(\mathrm{NO}_{3}\right)_{3} \bullet 9 \mathrm{H}_{2} \mathrm{O}\right)$ were sealed in a flask, slowly heated to $421 \mathrm{~K}$ and kept at this temperature while stirring for $16 \mathrm{~h}$. The crystalline material was filtrated and washed with DMF, methanol and acetone. All solvents were removed by keeping the sample at $393 \mathrm{~K}$ under vacuum for $2 \mathrm{~h}$. Powder XRD in air was carried out on a Siemens D5000 diffractometer equipped with a $\mathrm{Cu}$ anode $\mathrm{X}$-ray source (Cu K $\alpha, \lambda=1.5406 \AA)$.

\subsection{EPR measurements and analysis}

X-band EPR measurements were performed with a Bruker ESP300E spectrometer equipped with an ER 4102-ST rectangular cavity or an ER 4114HT high-temperature resonator, a HP5350 B frequency counter and a Bruker NMR ER 035M Gaussmeter, using diphenyl picrylhydrazyl (DPPH; $g=2.0036$ ) for field calibration. For presentation, all spectra are frequency normalized to $9.745 \mathrm{GHz}$. A Bruker ElexSys E500 spectrometer was used for the Qband EPR measurements, equipped with a CF910 He-flow Oxford Instruments cryostat (2- 
300 K). A Pendulum CNT-90XL frequency counter and a Bruker NMR ER 035M Gaussmeter were used to measure microwave frequency and magnetic field, respectively. The $g_{\perp}$ component of the $\mathrm{CO}_{3}{ }^{3-}$ radical in irradiated calcite powder ( $\mathrm{g}=2.0031$ ) [20] was used as a reference for field calibration. All spectra are normalized to $34 \mathrm{GHz}$ frequency. Spectra in both bands were recorded at RT, $5 \mathrm{~mW}$ microwave power, $100 \mathrm{kHz}$ modulation frequency and $0.2 \mathrm{mT}$ modulation amplitude. Simulations of EPR spectra were performed using the Easyspin toolbox (version 5.2.11) for Matlab [21].

For X-band EPR measurements, powder samples were placed in one-end closed quartz tubes, either in ambient air, evacuated by continuous pumping ( $p \approx 0.3 \mathrm{mbar}$ ), or exposed to pure oxygen gas. For $\mathrm{Q}$-band EPR measurements, powder samples were also placed in quartz tubes. Spectra were recorded either in air, under vacuum by continuously pumping the sample tube, or by flushing the cavity with He gas (N50: $\geq 99.99 \%$ mole). Under the latter two conditions, identical spectra were obtained. For the X-band measurements as a function of $\mathrm{O}_{2}$ pressure (Alphagaz $1 \mathrm{O}_{2}: \geq 99.995 \%$ mole), sample tubes were first evacuated and compressed $\mathrm{O}_{2}$ was then introduced in steps of approximately 50 mbar. After pressure equilibration, at least 15 minutes were waited before recording the EPR spectra.

\section{Results and discussion}

\subsection{X-band/Q-band EPR spectrum of DUT-5(Al)}

In Figure $2 \mathrm{~A}$ the X-band (left) and Q-band (right) RT EPR spectrum of DUT-5(Al), doped with $1 \% \mathrm{~V}$ is shown measured in vacuum $(p \approx 0.3 \mathrm{mbar})$ and in air. At this low $\mathrm{V}$ concentration, the broad EPR spectrum as found for COMOC-2(V) and MIL-47(V), due to strongly interacting $\mathrm{V}^{\mathrm{IV}}=\mathrm{O}$ ions, is nearly absent $[19,22]$. Instead, a spectrum typical of isolated vanadyl ions $\left(V^{I V}=0,3 d^{1}\right.$ configuration for $\left.V^{I V}, S=1 / 2, I\left({ }^{51} V\right)=7 / 2\right)$ with resolved ${ }^{51} \mathrm{~V}$ hyperfine (HF) structure $\left(A_{z} \approx 500 \mathrm{MHz}, A_{x} \approx A_{y} \approx 150 \mathrm{MHz}\right)$ is visible. The spectrum measured in air seems to be originating from just one component. However, when the spectrum is measured in vacuum, two components are clearly visible. The broad-line (BL) component, visible in both air and vacuum, features a large line width. The other component in vacuum exhibits much narrower lines, but its linewidth broadens so much in air that it becomes unresolvable from the $\mathbf{B L}$ component (see also 3.1.2).

\subsubsection{Narrow-line component}

Since the BL component is hardly affected by air exposure, it could be eliminated from the spectrum recorded in vacuum by subtraction of the $\mathbf{B L}$ spectrum measured in air. The remaining narrow-line component (further on labelled Ip) was then convincingly reproduced by a simulation using a single set of spin-Hamiltonian parameters (Table 1 ) as shown in Figure $2 \mathrm{~B}$. Simulations of the EPR spectra were performed by diagonalization of the spinHamiltonian

$$
\widehat{H}=\mu_{B} \hat{\vec{S}} \cdot \tilde{g} \cdot \vec{B}+\hat{\vec{I}} \cdot \tilde{A} \cdot \hat{\vec{S}}-g_{n} \mu_{N} \hat{\vec{I}} \cdot \vec{B}
$$

with $\mu_{B}$ and $\mu_{N}$ the Bohr and Nuclear magnetons. In Table 1 the principal values of the $\mathrm{g}$ tensor and the ${ }^{51} \mathrm{~V} \mathrm{HF}(\mathrm{A})$ tensor are shown, together with the relative Euler angles describing the tilting between the principal axes of both tensors. The experimental spectra 
of both bands were fitted simultaneously with the same spin-Hamiltonian parameters, allowing only for differences in the anisotropic Gaussian line broadening ("HStrain") between the two bands. Linewidth parameters used in the simulations are presented in the figure caption.

\begin{tabular}{|c|c|c|c|c|c|c|c|c|c|c|c|}
\hline Vanadyl centre & $g_{x}$ & $\mathrm{~g}_{\mathrm{y}}$ & $g_{z}$ & $A_{x}$ & $A_{y}$ & $A_{z}$ & $\alpha$ & $\beta$ & 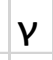 & $\delta g_{x y}$ & $\delta A_{x y}$ \\
\hline & & & & $\mathrm{MHz}$ & $\mathrm{MHz}$ & $\mathrm{MHz}$ & $\circ$ & $\circ$ & $\circ$ & $\times 10^{4}$ & $\mathrm{MHz}$ \\
\hline MIL-53(Al) np-h 1 & 1.9722 & 1.9623 & 1.9316 & 164.9 & 174.2 & 506.4 & -8 & -14 & 0 & 99 & -9.3 \\
\hline MIL-53(AI) np-h 2 & 1.9713 & 1.9594 & 1.9324 & 162.5 & 170.9 & 504.1 & -8 & -14 & 0 & 119 & -8.4 \\
\hline MIL-53(AI) np-d & 1.9719 & 1.9634 & 1.9292 & 165.9 & 173.0 & 505.7 & -8 & -14 & 0 & 85 & -7.1 \\
\hline MIL-53(Al) as & 1.9725 & 1.9669 & 1.9391 & 163.0 & 165.0 & 492.0 & -8 & -12 & 0 & 56 & -2.0 \\
\hline MIL-53(AI) Ip & 1.9724 & 1.9675 & 1.9387 & 165.6 & 164.3 & 495.5 & 0 & -11 & 0 & 49 & 1.3 \\
\hline DUT-5(AI) Ip & 1.9729 & 1.9685 & 1.9391 & 167.3 & 165.7 & 495.5 & 0 & -10 & 0 & 44 & 1.5 \\
\hline DUT-5(AI) BL & 1.9760 & 1.9701 & 1.9408 & 184.8 & 154.7 & 509.3 & 0 & 0 & 0 & 59 & 30 \\
\hline
\end{tabular}

Table 1: Spin-Hamiltonian parameter set extracted from $X$ - and $Q$-band EPR spectra at RT of $V$-doped MIL-53(Al) and V-doped DUT-5(Al). Principal values of the $g$ tensor and the ${ }^{51} \mathrm{VHF}$ (A) tensor are shown, together with the relative Euler angles describing the tilting between the principal axes of both tensors (for their definition, see ref. [21]) and a comparison of the difference between the $x$ - and $y$-components of the $g$ and ${ }^{51} V A$ tensors. The parameters for the four MIL-53(Al) states are reproduced from Nevjestić et al. [14] (tilting angles are adjusted to the Easyspin convention of version 5.2.11). Errors on the principal values for the DUT-5(Al) components: Ip $\Delta g= \pm 0.0005, \Delta A= \pm 3 \mathrm{MHz}$ and $\Delta \alpha, \Delta \beta, \Delta \gamma= \pm 1^{\circ} ; \mathbf{B L}$ : $\Delta g_{x, z}= \pm 0.0010, \Delta g_{y}= \pm 0.0020, \Delta A= \pm 5 \mathrm{MHz}$ and $\Delta \alpha, \Delta \beta, \Delta \gamma= \pm 5^{\circ}$. 

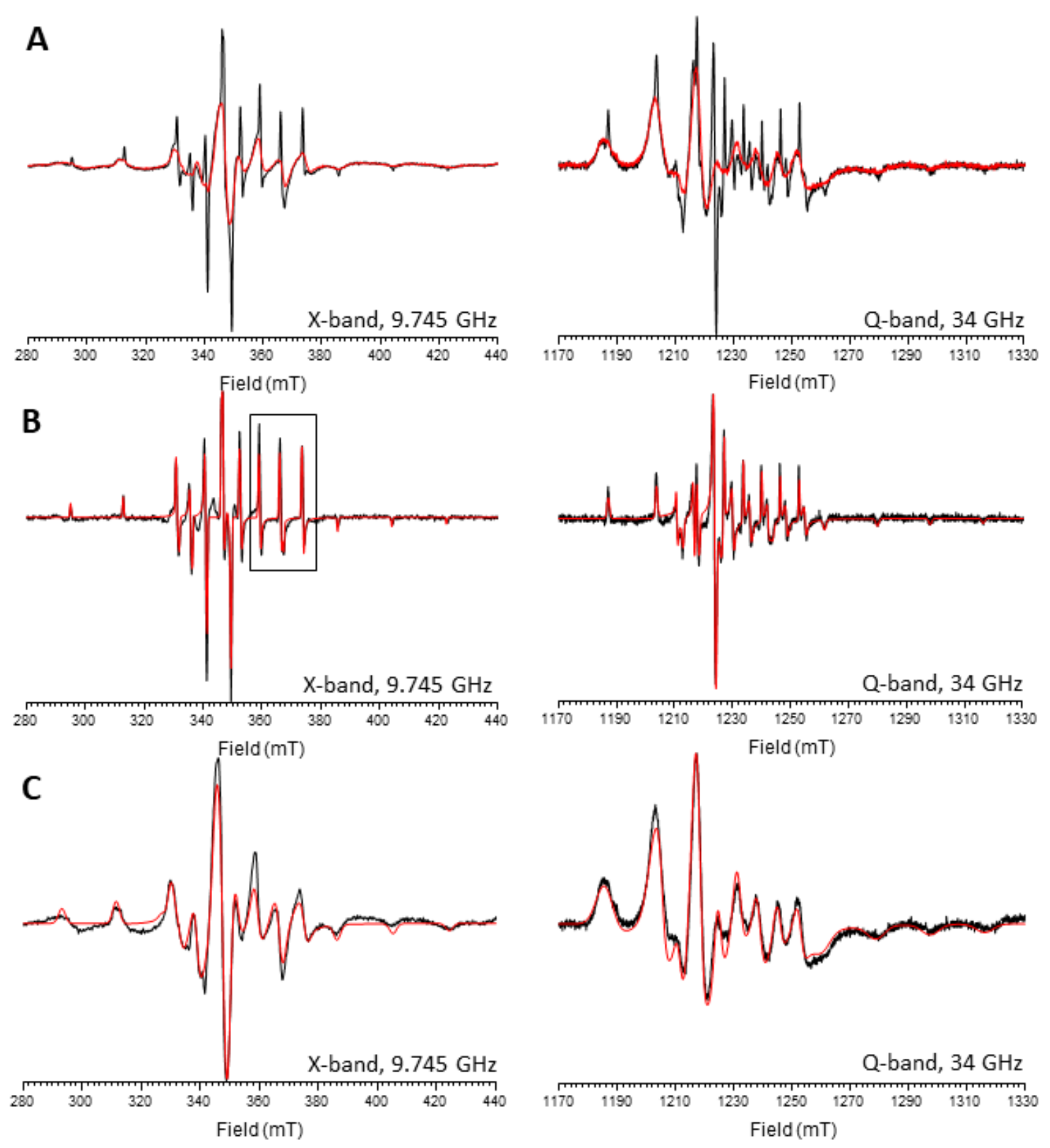

Figure 2: X-band (left, $9.745 \mathrm{GHz}$ ) and Q-band (right, $34 \mathrm{GHz}$ ) RT powder EPR spectra of: A: DUT-5(Al), doped with $1 \% \mathrm{~V}$, measured in vacuum ( $p \approx 0.3 \mathrm{mbar}$ ) - black curves, and under ambient air pressure - red curves. B: the Ip component of DUT-5(Al), doped with $1 \% \mathrm{~V}$, obtained by elimination of the BL component from the spectra in A: black - experiment; red - simulation. An anisotropic Gaussian linewidth was used for the simulations (Xband: HStrain $=[14,14,14] \mathrm{MHz}$; Q-band: HStrain $=[13,23,31] \mathrm{MHz})$. The black box indicates the zoomed-in region shown in Figure 3 B. C: the BL component of DUT-5(AI) (spectra recorded in air, from A), doped with 1\% $V$ : black - experiment, red - simulation. An anisotropic Gaussian linewidth was used for the simulations (X-band: HStrain $=[71,113,74] \mathrm{MHz}$; Q-band: HStrain $=[74,246,196] \mathrm{MHz})$.

As mentioned earlier by Depauw et al. [19], the characteristics of the spectrum of the narrow-line component are very similar to those of the spectrum of V-doped MIL-53(AI) in the Ip state. This similarity, including the line broadening upon exposure to air $\left(\mathrm{O}_{2}\right.$, see 3.1.2), leaves little doubt that this center corresponds with $\mathrm{V}^{\mathrm{IV}}=\mathrm{O}$ substituting for $\mathrm{Al}-\mathrm{OH}$ in DUT-5(AI), which was reported to only occur in an open pore (Ip) state [12]. Therefore we 
will label this narrow-line component as Ip from here on. Most of the Ip features are very well reproduced by the simulations in Figure $2 \mathrm{~B}$, both in position and in relative line intensity, certainly considering the presence of the $\mathbf{B L}$ component in the original spectra.

\subsubsection{Oxygen pressure dependence of the Ip component}

As mentioned before, the spectrum of the Ip component of DUT-5(Al) increases in linewidth with increasing air pressure (Figure 3). As for the Ip state in MIL-53(Al), it is the interaction with $\mathrm{O}_{2}$ that produces this line broadening. For evaluation of the line broadening as a function of the $\mathrm{O}_{2}$ pressure for this spectrum, only part of it was recorded, as indicated in Figure $2 \mathrm{~B}$, and simulated, using the $\mathrm{g}$ and $\mathrm{A}$ parameters in Table 1 . The line shape also changed in this section from dominant Gaussian to Lorentzian. For the sake of consistency, all linewidth fittings assumed a Lorentzian line shape. In order to isolate the Ip component, from all spectra the spectrum recorded at 1 bar $\mathrm{O}_{2}$ was subtracted. For $\mathrm{O}_{2}$ pressures larger than 300 mbar, noise levels for the Ip component spectrum become very high, impeding accurate determination of the linewidth.

In analogy with the results for MIL-53(AI), the EPR linewidth increases close to linearly with oxygen pressure in the low pressure range. In fact, even in absolute values the $\mathrm{O}_{2}$ pressure dependence of the linewidth closely follows that for the MIL-53(AI) Ip state [14], as illustrated in Figure $3 \mathrm{~A}$. In view of the differences in the pore size between MIL-53(AI) and DUT-5(Al), this was not a priori expected. Thus the Ip component of DUT-5(Al) as oxygen sensor is equally sensitive as $\mathrm{V}^{\mathrm{IV}}=\mathrm{O}$ in the Ip state of MIL-53(Al), and has the advantage of not transforming to a $\mathrm{np}$ state at ambient pressure and humidity conditions. However, the major disadvantage is the presence of the BL component, which makes linewidth evaluation more difficult.

A

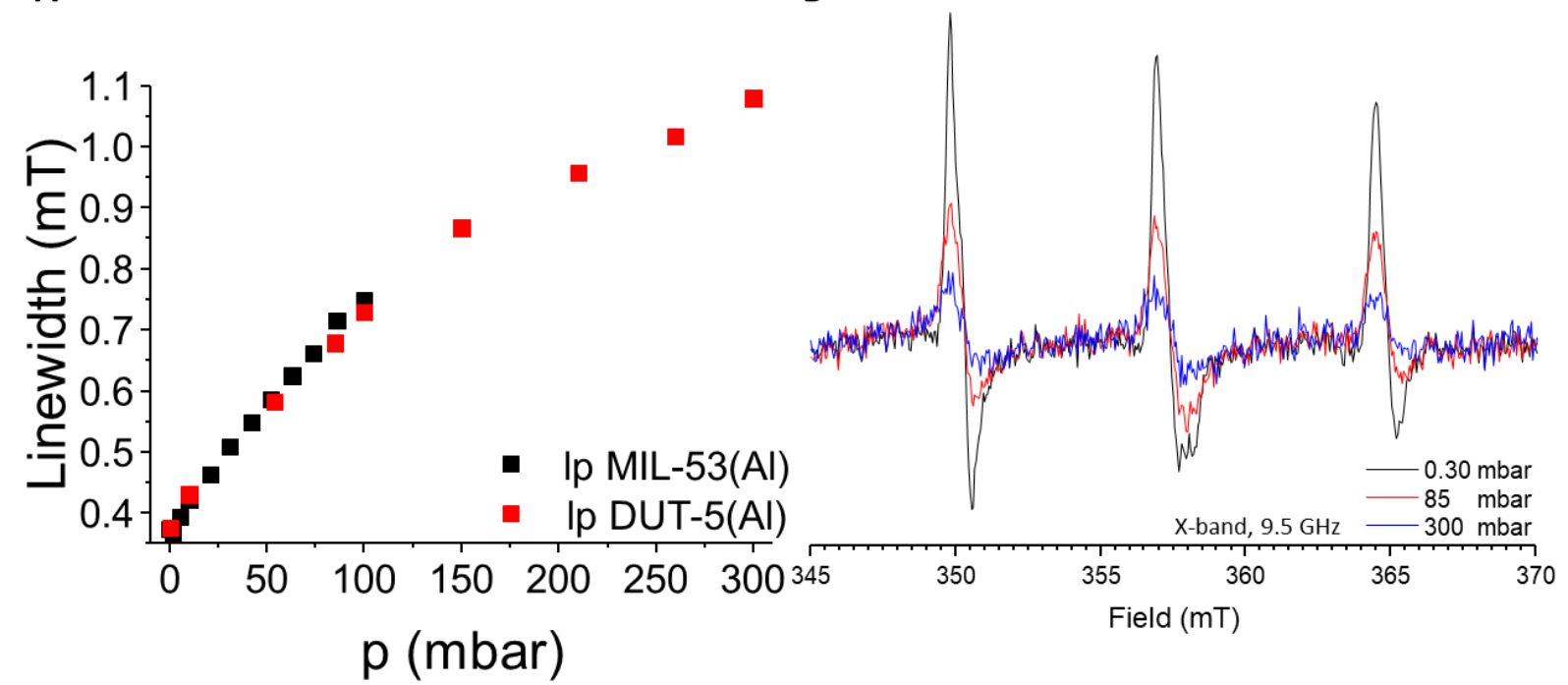

Figure 3: A: Comparison of the linewidth increase of the spectra of both the 1\% V-doped DUT-5(AI) Ip component and the $1 \% \mathrm{~V}$-doped MIL-53(Al) Ip state [14] in function of $\mathrm{O}_{2}$ pressure. B: Experimental X-band (9.5 $\mathrm{GHz}) R T$ powder EPR spectra of the Ip component of DUT-5(Al), zoomed in as shown in Figure 2 B. The spectrum of the BL component has been subtracted from the spectrum measured in vacuum. 


\subsubsection{Broad-line component}

Figure $2 \mathrm{C}$ shows the $\mathrm{X}$ - and Q-band experimental spectra of $1 \% \mathrm{~V}$-doped DUT-5(Al) measured in air, containing (mainly) the BL component, and their best-fit simulation. The spin-Hamiltonian parameters used are shown in Table 1. An anisotropic Gaussian linewidth was used in both bands. All features in the simulations are at the correct field positions. However, especially in the X-band spectrum, the reproduction of the relative line intensities is still somewhat problematic. This definitely indicates that the errors on the obtained parameters are larger than for the Ip spectrum. In addition, a more sophisticated line broadening model, including strain on the $g$ and A parameters, might be necessary. In view of the limited experimental data available (spectra in only two bands) and a possible (slight) influence of the broadened Ip component, such simulations have at this moment not yet been attempted. We checked through fitting and simulation that the BL spectrum cannot be reproduced with the same spin-Hamiltonian parameters of the Ip component by solely fitting the linewidth.

For identifying the $\mathbf{B L}\left(\mathrm{V}^{\mathrm{I}}=\mathrm{O}\right)$ component a comparison between the spin-Hamiltonian parameters in Table 1 may provide insight. As already discussed by Nevjestić et al. [13], the difference between $g_{x}$ and $g_{y}$ reflects the larger deviation from axial symmetry for the $\mathbf{n p}$ states as compared to the Ip and as states for V-doped MIL-53(Al). This also results in a larger number of resolved spectral features in the corresponding spectra. In addition, the $g_{z}$ parameter is around 1.93 for the spectra of the np states of MIL-53(Al), while closer to 1.94 for the spectra of the Ip and as states. Both components of DUT-5(AI) have a near-axial symmetry with a difference between the $g_{x}$ and $g_{y}$ values comparable to the $I p$ and as state of MIL-53(Al) and a $g_{z}$ value close to 1.94. From the perspective of their $g$ tensor, both are thus open-pore-like. Another remarkable difference is found in the $A_{z} H F$ parameter, which is about $10 \mathrm{MHz}$ larger for the $\mathbf{n p}$ states in MIL-53(Al), as compared to the Ip and as states. This is also about the difference between the $A_{z}$ HF parameters of both components in DUT-5(AI). The $\beta$ angle is largest for the spectra of the MIL-53(AI) np states and becomes smaller for the open-pore-like states. The principal axes of the $g$ and ${ }^{51} \mathrm{~V} A$ tensor of the $\mathbf{B L}$ component in DUT-5(Al) are even not mutually tilted! While the spectrum of the Ip component in DUT5(Al) is exclusively related to the spectrum of the Ip state of MIL-53(Al), the spectrum of the BL component in DUT-5(Al) seems to adopt characteristics of the spectra of both states. So its identification is definitely not so obvious.

Moreover, the deviation in spin-Hamiltonian parameters between this BL component and the Ip component is larger than between the as and Ip states, or between the $\mathbf{n p}-\mathbf{h}$ and $\mathbf{n p}-\mathbf{d}$ states in MIL-53(Al). This points to a more severe structural difference between this BL component and the Ip component than a difference in pore filling and even than the broadening by residual paramagnetic gas. One possible model for this center is that the vanadyl is located at a defect site, e.g. trapped in the framework pores or incorporated at a broken linker metal site. The larger linewidth may then result from additional (vibrational) degrees of freedom that such vanadyl ions exhibit. Another possible model is that this vanadyl center is more free, located at the surface of the framework, or inside the pores/grains, more comparable with unbound vanadium complexes. This could explain the 
lack of tilting between the principal axes of $g$ and $A$. This possibility has been investigated by Nevjestić [23]. $\mathrm{VOSO}_{4}$ was inserted into the pores of DUT-5(Al) by mixing $1 \%$ of a $\mathrm{VOSO}_{4}$ solution with $99 \%$ of the powder, stirring for 10 minutes at $421 \mathrm{~K}$. In these conditions we do not expect the incorporation of $\mathrm{V}^{\mathrm{IV}}$ inside of the framework, but rather that it would be dispersed in the pores. Measurements in X-band revealed a $\mathrm{V}^{\mathrm{IV}}=\mathrm{O}$ related EPR spectrum, with large linewidths like the BL spectrum, but with clearly different HF structure. Additional research will thus be necessary to elucidate the model for the $\mathbf{B L}$ center.

\subsection{Concentration series}

Figures $4 \mathrm{~A}$ and $\mathrm{B}$ show Q-band spectra of DUT-5(AI) doped with different concentrations of $\mathrm{V}^{\mathrm{IV}}$, recorded at RT in helium atmosphere. At low concentrations, there are the two components visible as discussed in the previous sections, both growing in intensity with increasing $\mathrm{V}^{\mathrm{IV}}$ concentration. The broad feature related to COMOC-2(V) [18] (see Figure $4 \mathrm{C}$ ) appears from $7 \% \mathrm{~V}^{\mathrm{IV}}$ and steadily grows with increasing $\mathrm{V}^{\mathrm{IV}}$ concentration. As a result of the strong interactions between $\mathrm{V}^{\mathrm{IV}}=\mathrm{O}$ ions along the metal-inorganic chains, no HF structure is resolved for this component. A simulation using an axial $g$ tensor $\left(g / /=1.957 ; g_{\perp}=1.968\right)$ is also shown in Figure $4 \mathrm{C}$.

As the $\mathrm{V}^{\mathrm{IV}}$ concentration increases, extra structure appears in the spectrum as indicated by the arrows in Figure $4 \mathrm{~A}$, due to the $z$-component (smallest principal $g$ and largest principal A) HF pattern of an additional vanadyl center. The central part ( $x$ and $y$ components) of this spectrum is not resolved. The extra pattern is first clearly observed in the spectrum of the sample with $9 \% \mathrm{~V}$, and is most prominent for the $23 \% \mathrm{~V}$ sample. This was also confirmed by re-examination of the X-band spectra presented in ref. [23, 24]. 

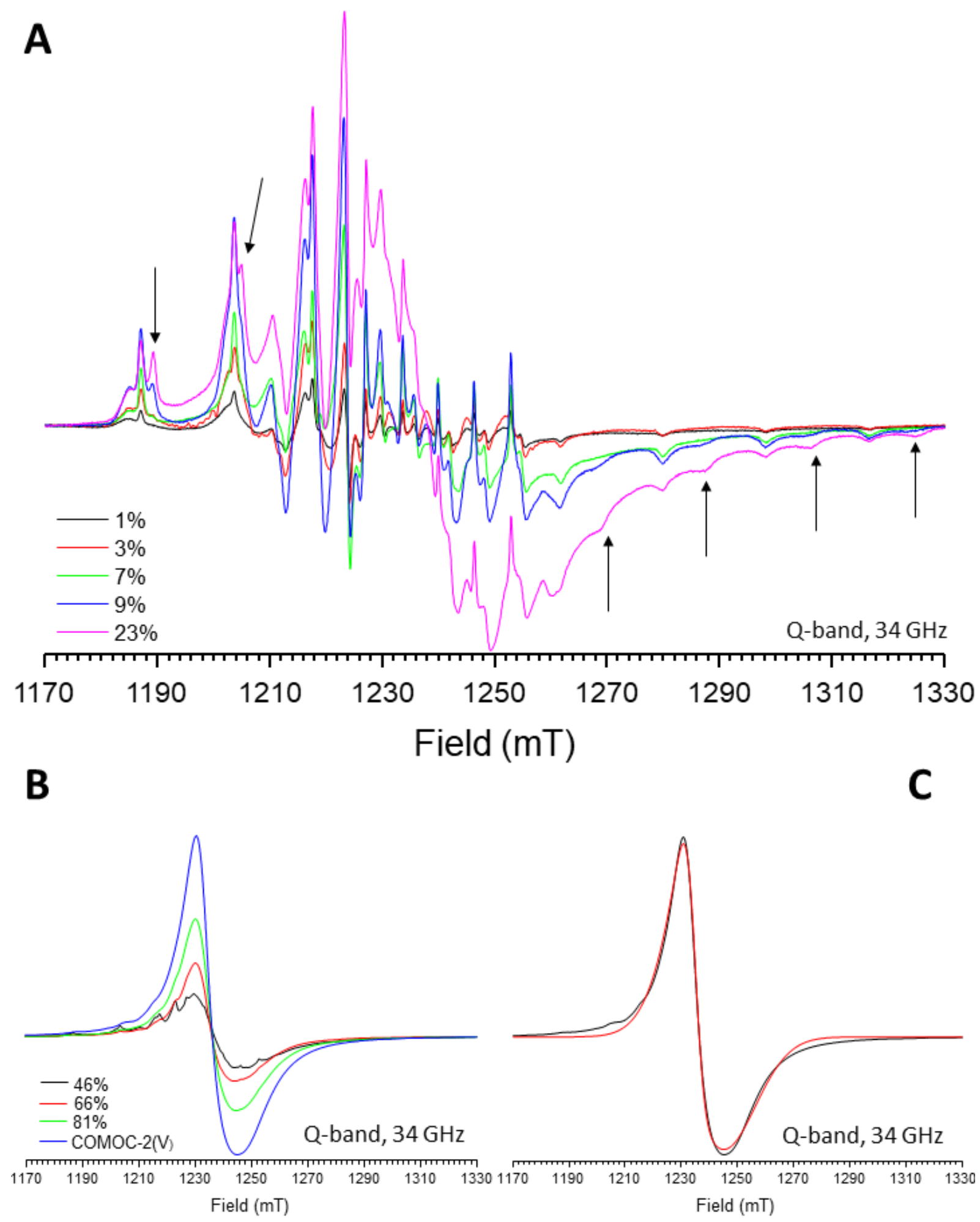

Figure 4: Experimental Q-band (34 GHz) RT powder EPR spectra of A: DUT-5(Al), doped with increasing (1\% to 23\%) concentrations of $V^{I V}$. Arrows indicate the additional features attributed to $V^{I V}=O$ in the np framework state. B: DUT-5(Al), doped with increasing (46\% to 81\%) concentrations of $V^{\prime V}$ and COMOC-2(V) C: COMOC-2(V). Black - experiment, red - simulation with $g_{/ /}=1.957$ and $g_{\perp}=1.968$. The spectra are normalized in height.

Simulating the spectrum of the np states of MIL-53(Al) and comparing with the X-and Qband spectra of $23 \% \mathrm{~V}$-doped DUT-5(Al), showed very satisfactory agreement with the zcomponent HF lines. This is illustrated with the simulation of the $\mathbf{n p}-\mathbf{d}$ state in Figure $5 \mathrm{~A}$. 
One would thus be tempted to identify this component as $\mathrm{V}^{\mathrm{IV}}=\mathrm{O}$ incorporated in the metal nodes of a $\mathbf{n p}$ state of the mixed-metal MOF. This is not a priori expected, since no np state has been reported for the rigid DUT-5(Al) framework. Nevertheless, our previous high resolution synchrotron XRD study of mixed $(\mathrm{Al}-\mathrm{OH})_{1-\mathrm{x}}(\mathrm{V}=\mathrm{O})_{\times} \mathrm{BPDC}$ frameworks [19] has shown that for samples with $46 \% \mathrm{~V}$ concentration and more, at $233 \mathrm{~K}$ and low pressure, the Ip and np framework states coexist.

A re-examination of the XRD patterns (Figure $5 \mathrm{~B}$ ) for the samples with low $\mathrm{V}$ concentration reveals that for the $9 \% \mathrm{~V}$ and $23 \% \mathrm{~V}$ samples clear shoulders on the Ip state peaks can be seen that can be linked to a np state, in agreement with the EPR results. This leads to the tentative conclusion that even fairly low global concentrations of $\mathrm{V}$ are able to induce framework flexibility of the rigid DUT-5(Al). Further spectroscopic and electron microscopic research will, however, be necessary to confirm this hypothesis and to understand this remarkable impact of metal mixing.
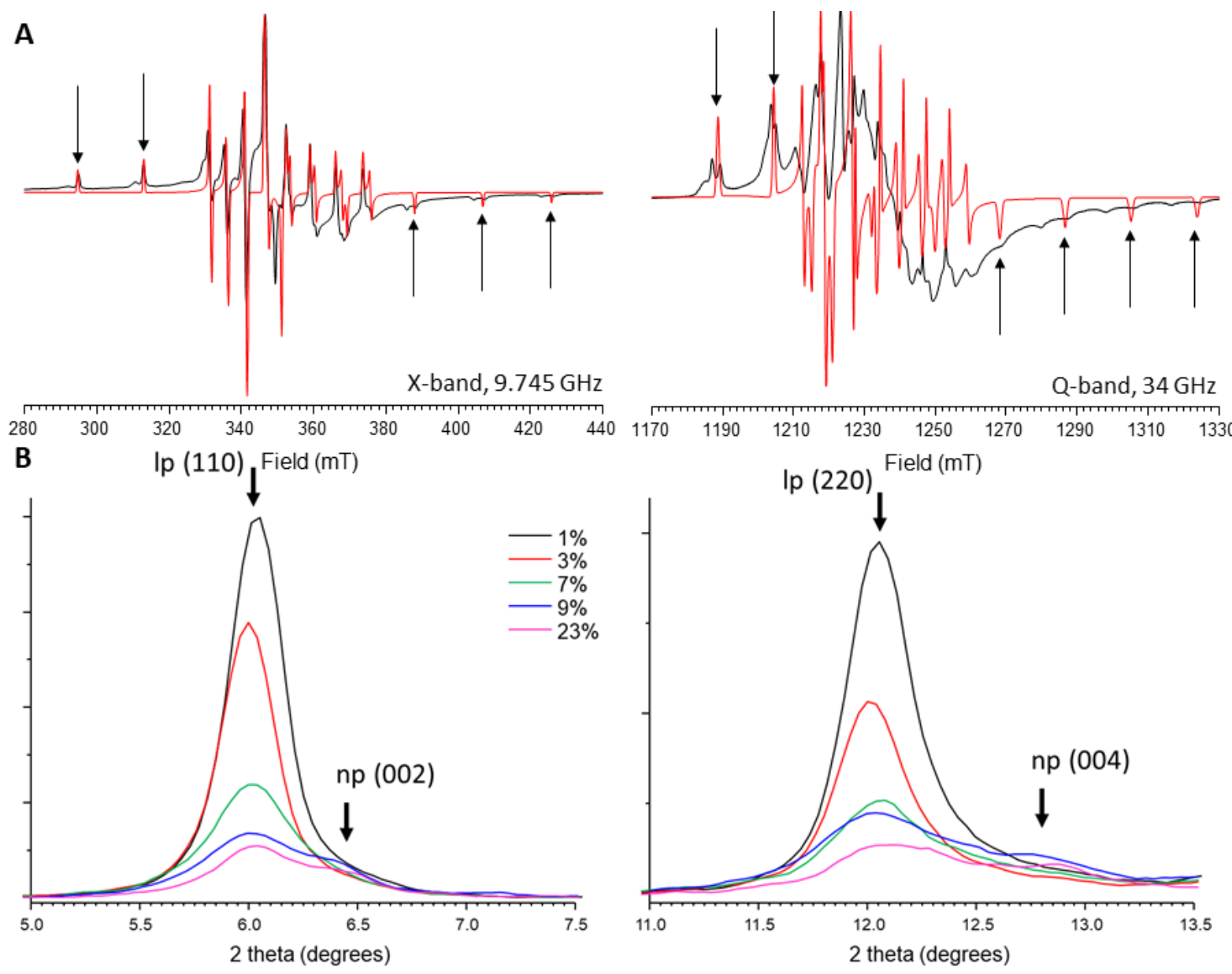

Figure 5: A: Black - Experimental X-band (left, $9.745 \mathrm{GHz}$ ) and Q-band (right, $34 \mathrm{GHz}$ ) RT powder EPR spectra of $23 \%$ V-doped DUT-5(Al). Red-X-band (left) and Q-band (right) simulation of the MIL-53(Al) np-d state spectrum. Arrows indicate the z-component HF pattern lines. $B: V$ concentration dependence of XRD patterns of DUT-5(Al) in air in two different $2 \vartheta$ regions, $\lambda=1.54056 \AA$. Miller indices for the peaks related to $\mathbf{p}$ and $\boldsymbol{n} \boldsymbol{p}$ are indicated; $\boldsymbol{n p}(002)$ peak assignments have been made based on similarity with the COMOC-2(V) patterns. 


\section{Conclusions}

Doping DUT-5(Al) with low (1\% - 7\%) concentrations of $\mathrm{V}^{\mathrm{IV}}$ reveals two components in the EPR spectrum measured in vacuum: a Ip and a BL component. Both have a spectrum typical for well dispersed vanadyl ions. The Ip component has spin-Hamiltonian parameters nearly identical to those of the Ip state of MIL-53(Al). It also exhibits EPR linewidth broadening with oxygen pressure and is equally sensitive as $V^{I V}=0$ in the Ip state of MIL-53(Al). The EPR spectrum of the BL component has characteristics of both $\mathbf{n p}$ and Ip states of MIL-53(AI). Additional research will be necessary to identify this component. Increasing the concentration of $\mathrm{V}^{\mathrm{IV}}$ in the framework of DUT-5(AI) reveals additional spectral components. A broad single line without HF structure, similar to the spectrum of COMOC-2(V), steadily grows with increasing $\mathrm{V}^{\mathrm{IV}}$ concentration. Secondly, the z-component HF pattern of an additional isolated vanadyl center is observed. Comparison with MIL-53(Al) points to a $\mathbf{n p}$ state in DUT-5(AI). A re-examination of the XRD patterns for these samples supports the hypothesis of the presence of a np state even at fairly low (9\%) global V concentrations.

\section{Acknowledgements}

The authors gratefully thank the Research Foundation Flanders (FWO-Vlaanderen) for financially supporting this work (Grant No. G0048.13N).

\section{References}

1. Hu, Z., B.J. Deibert, and J. Li, Luminescent Metal-Organic Frameworks for Chemical Sensing and Explosive Detection. Chemical Society Reviews, 2014. 43(16): p. 5815-5840.

2. Leus, K., et al., The remarkable catalytic activity of the saturated metal organic framework VMIL-47 in the cyclohexene oxidation. Chemical Communications, 2010. 46(28): p. 5085-5087.

3. Li, J.-R., R.J. Kuppler, and H.-C. Zhou, Selective Gas Adsorption and Separation in MetalOrganic Frameworks. Chemical Society Reviews, 2009. 38(5): p. 1477-1504.

4. Kreno, L.E., et al., Metal-Organic Framework Materials as Chemical Sensors. Chemical Reviews, 2012. 112(2): p. 1105-1125.

5. Loiseau, T., et al., A Rationale for the Large Breathing of the Porous Aluminum Terephthalate (MIL-53) Upon Hydration. Chemistry-a European Journal, 2004. 10(6): p. 1373-1382.

6. Volkringer, C., et al., XRD and IR Structural Investigations of a Particular Breathing Effect in the MOF-type Gallium Terephthalate MIL-53(Ga). Dalton Transactions, 2009(12): p. 22412249.

7. Llewellyn, P.L., et al., Complex Adsorption of Short Linear Alkanes in the Flexible MetalOrganic-Framework MIL-53(Fe). Journal of the American Chemical Society, 2009. 131(36): p. 13002-13008.

8. Serre, C., et al., Very Large Breathing Effect in the First Nanoporous Chromium(III)-Based Solids: MIL-53 or $\mathrm{Cr}^{\prime \prime \prime}(\mathrm{OH})\left\{\mathrm{O}_{2} \mathrm{C}-\mathrm{C}_{6} \mathrm{H}_{4}-\mathrm{CO}_{2}\right\}\left\{\mathrm{HO}_{2} \mathrm{C}-\mathrm{C}_{6} \mathrm{H}_{4}-\mathrm{CO}_{2} \mathrm{H}_{x} \mathrm{H}_{2} \mathrm{O}_{y}\right.$. Journal of the American Chemical Society, 2002. 124(45): p. 13519-13526.

9. Millange, F., et al., Effect of the Nature of the Metal on the Breathing Steps in MOFs with Dynamic Frameworks. Chemical Communications, 2008(39): p. 4732-4734.

10. Mowat, J.P.S., et al., A Novel Structural Form of MIL-53 Observed for the Scandium Analogue and its Response to Temperature Variation and $\mathrm{CO}_{2}$ Adsorption. Dalton Transactions, 2012. 41(14): p. 3937-3941.

11. Barthelet, K., et al., A breathing hybrid organic-inorganic solid with very large pores and high magnetic characteristics. Angewandte Chemie-International Edition, 2002. 41(2): p. 281. 
12. Nevjestic, I., et al., Multi-frequency (S, X, Q and W-band) EPR and ENDOR Study of Vanadium(IV) Incorporation in the Aluminium Metal-Organic Framework MIL-53. Chemphyschem, 2015. 16(14): p. 2968-73.

13. Nevjestić, I., et al., In Situ Electron Paramagnetic Resonance and X-ray Diffraction Monitoring of Temperature-Induced Breathing and Related Structural Transformations in Activated VDoped MIL-53(Al). The Journal of Physical Chemistry C, 2016. 120(31): p. 17400-17407.

14. Nevjestic, I., et al., Sensing the framework state and guest molecules in MIL-53(Al) via the electron paramagnetic resonance spectrum of V(IV) dopant ions. Phys Chem Chem Phys, 2017. 19(36): p. 24545-24554.

15. Bignami, G.P.M., et al., Cost-effective (17)O enrichment and NMR spectroscopy of mixedmetal terephthalate metal-organic frameworks. Chem Sci, 2018. 9(4): p. 850-859.

16. Ortiz, G., et al., New Insights into the Hydrogen Bond Network in Al-MIL-53 and Ga-MIL-53. The Journal of Physical Chemistry C, 2014. 118(38): p. 22021-22029.

17. Senkovska, I., et al., New highly porous aluminium based metal-organic frameworks: $\mathrm{Al}(\mathrm{OH})(\mathrm{ndc})$ ( $n d c=2,6$-naphthalene dicarboxylate) and $\mathrm{Al}(\mathrm{OH})(\mathrm{bpdc})\left(\mathrm{bpdc}=4,4^{\prime}\right.$-biphenyl dicarboxylate). Microporous and Mesoporous Materials, 2009. 122(1-3): p. 93-98.

18. Liu, Y.Y., et al., New V(IV)-based metal-organic framework having framework flexibility and high CO2 adsorption capacity. Inorg Chem, 2013. 52(1): p. 113-20.

19. Depauw, H., et al., Discovery of a novel, large pore phase in a bimetallic Al/V metal-organic framework. Journal of Materials Chemistry A, 2017. 5(47): p. 24580-24584.

20. Serway, R.A. and S.A. Marshall, Electron Spin Resonance Absorption Spectra of $\mathrm{CO}_{3}{ }^{-}$and $\mathrm{CO}_{3}{ }^{3-}$ Molecule-lons in Irradiated Single-Crystal Calcite. The Journal of Chemical Physics, 1967. 46(5): p. 1949-1952.

21. Stoll, S. and A. Schweiger, EasySpin, a Comprehensive Software Package for Spectral Simulation and Analysis in EPR. Journal of Magnetic Resonance, 2006. 178(1): p. 42-55.

22. Leus, K., et al., The Coordinatively Saturated Vanadium MIL-47 as a Low Leaching Heterogeneous Catalyst in the Oxidation of Cyclohexene. Journal of Catalysis, 2012. 285(1): p. 196-207.

23. Nevjestic, I., In Situ Electron Paramagnetic Resonance Study of Breathing Phenomena and Related Framework Transformations in Paramagnetically Doped Metal-organic Frameworks, in Faculty of Sciences. 2018, Ghent University: Ghent, Belgium.

24. Depauw, H., Investigation of the Doping Effect in Bimetallic Metal-organic Frameworks to Tune Their Structural Flexibility, in Faculty of Sciences. 2018, Ghent University: Ghent, Belgium. 